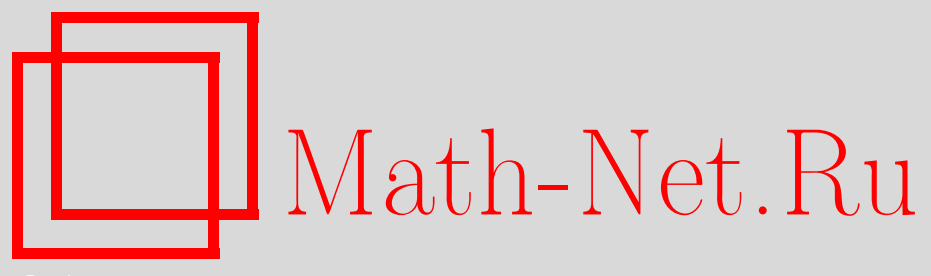

М.-М. Деза, М. И. Штогрин, Типы и граничная единственность полипентагонов, УМН, 2006, том 61, выпуск $6,183-184$

DOI: https://doi.org/10.4213/rm4488

Использование Общероссийского математического портала Math-Net.Ru подразумевает, что вы прочитали и согласны с пользовательским соглашением http://www . mathnet.ru/rus/agreement

Параметры загрузки:

IP : 35.173 .219 .149

26 апреля 2023 г., 13:29:07

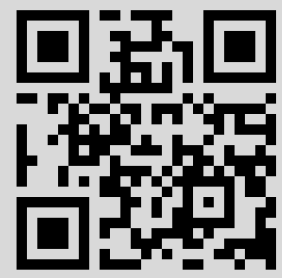




\title{
Типы и граничная единственность полипентагонов
}

\author{
М. Деза, М. И. Штогрин
}

Неразделимый плоский граф, взятый вместе с внутренними гранями, образует $(r, q)$-полицикл, если все внутренние грани суть $r$-угольники (где $r=$ const $\geqslant 3$ ), а все внутренние вершины имеют наибольшую из степеней, обозначаемую через $q$ (где $q=$ const $\geqslant 3)$, см. [1]. Каждый конечный $(r, q)$-полицикл гомеоморфен кругу, а его край гомеоморфен окружности. Циклическую последовательность $q_{1} q_{2} \ldots q_{m}$, составленную из степеней $q_{i}, i=1, \ldots, m$, всех $m$ вершин края $(r, q)$-полицикла, назовем граничным кодом ${ }^{1}$. Назовем $r$-угольник приграничным, если хотя бы одно его ребро принадлежит краю. Если пересечение приграничного $r$-угольника с краем является связным, то он устраним - без этого $r$-угольника остаток снова является $(r, q)$-полициклом, см. [2]. Два $(r, q)$-полицикла с одним и тем же краем назовем эквиграничными. Эквиграничные $(r, q)$-полициклы состоят из одного и того же числа $r$-угольников $p_{r}$, см. [3]. Эквиграничную пару $(r, q)$-полициклов назовем неприводимой, если каждое ребро края, принадлежащее устранимому $r$-угольнику одного $(r, q)$-полицикла из данной пары, принадлежит неустранимому $r$-угольнику другого $(r, q)$-полицикла из этой же пары, см. [2]. Для каждого $r \geqslant 5$ в [4] найден пример эквиграничной пары $(r, 3)$-полициклов с числом $p_{r}=4 r$ и высказана гипотеза, что $4 r$ является минимальным значением для величины $p_{r}$. Эта гипотеза доказана в [2] при $r=6$. Ниже она доказана при $r=5$, т.е. для $(5,3)$-полициклов; назовем их полипентагонами ${ }^{2}$.

Теорема. (i) При $p_{5} \leqslant 19$ нет ни одной пары эквиграничных полипентагонов.

(ii) При $p_{5}=20$ существует одна (неприводимая) пара эквиграничных полипентагонов. Она имеет следующий граничный код: $3^{3} 232323^{6} 232323^{3} 232323^{6} 23232$.

(iii) При $p_{5}=21$ имеются три (приводимье) пары. $3^{3} 232323^{6} 2323^{4} 2323^{6} 23232$, $3^{3} 232323^{6} 23^{2} 2^{2} 3^{4} 232323^{6} 23232,3^{3} 232323^{7} 2^{2} 3^{2} 23^{3} 232323^{6} 23232$ - их граничные коды.

(iv) При $p_{5}=22$ имеется семнади,ть пар эквиграничных полипентагонов.

(v) При любом $p_{5} \geqslant 23$ имеется не менее 17 пар, получаемых расширениями ${ }^{3}$.

Внутреннее ребро произвольного $(r, q)$-полицикла, чьи оба конца лежат на границе, назовем сквозным. Любой $(r, q)$-полицикл без сквозных ребер назовем элементарным.

ЛЕмма 1. Произвольный $(r, q)$-полииикл при разрезании по всем имеющимся у него сквозным ребрам однозначно разбивается на элементарные полициклы.

Эти элементарные полициклы назовем элементарными слагаемыми $(r, q)$-полицикла. Вид и обозначения элементарных слагаемых полипентагонов см. в [1; рис. 5].

Построим граф смежности элементарных слагаемых $(r, q)$-полицикла: вершины графа соответствуют элементарным слагаемым; две вершины графа соединены ребром, если соответствующие элементарные слагаемые смежны, т.е. имеют общее ребро.

ЛЕмма 2. Граф смежности элементарных слагаемых полииикла есть дерево.

В любом нетривиальном дереве склеек элементарных слагаемых содержатся по меньшей мере два висячих элементарных слагаемых. Одно граничное ребро висячего элементарного слагаемого является сквозным ребром объемлющего $(r, q)$-полицикла; все остальные его граничные ребра остаются граничными ребрами полицикла.

Граничный код $(r, q)$-полицикла мы можем записывать не только в развернутом виде, но и в свернутом виде, когда количество подряд идущих в циклической после-

Работа выполнена при финансовой поддержке Российского фонда фундаментальных исследований (грант № 05-01-00170), программы "Ведущие научные школы" (грант НШ4182.2006.1) и программы ОМН РАН "Современные проблемы теоретической математики".

1 Это вершинный граничный код. В работе [2] использован реберный граничный код.

${ }^{2}$ Известен пример граничного кода [4; предложение 3], допускающего ровно $n+1$ эквиграничных полипентагонов. Любой имеет $40 n+18$ вершин, $60 n+23$ ребер, $20 n+6$ граней.

${ }^{3}$ Если граничный код конечного полипентагона отличен от $3^{5}$, то он расширяем, см. [1]. 
довательности одинаковых степеней указывается в показателе. В случае полипентагона, т.е. (5,3)-полицикла, граничный код в свернутом виде состоит из двоек и троек с какими-то показателями. Набор показателей для двоек мы будем называть типом полипентагона и записывать в фигурных скобках. Так, например, в формулировке теоремы, случай (iii), полипентагоны с первым граничным кодом имеют тип $\{1\}$, а полипентагоны с двумя другими граничными кодами имеют тип $\{1 ; 2\}$ или $\{2 ; 1\}$.

Bсе (конечные и бесконечные) полипентагоны однозначно разбиваются на 9 типов.

Tun $\{5\}$. Единственным полипентагоном типа $\{5\}$ является $D$, см. [1; рис. 5].

Tun $\{3\}$. Любой полипентагон типа $\{3\}$ неэлементарен и состоит из элементарных слагаемых $C_{1}$ (каждое $C_{1}$ смежно с двумя соседними элементарными слагаемыми), $C_{3}, E_{1}$ (каждое $C_{3}, E_{1}$ смежно с тремя соседними элементарными слагаемыми), $D$ (каждое $D$ висячее: оно смежно с одним соседом). (Бес)конечные полипентагоны типа $\{1\}$ содержат не менее (одного) двух элементарных слагаемых $D$.

Tun $\{2\}$. Полипентагоны типа $\{2\}$ состоят из элементарных слагаемых $C_{1}, C_{3}, E_{1}$.

Tun $\{1\}$. Элементарные полипентагоны типа $\{1\}$ суть $A_{2}, A_{3}, A_{4}, A_{5}$. Неэлементарные полипентагоны типа $\{1\}$ состоят из элементарных слагаемых $C_{3}, E_{1}$ (смежных с 3 соседями), $C_{1}, C_{2}, D, E_{j}, j \geqslant 2$ (смежных с 2 соседями), $B_{1}, B_{2}, B_{3}$ (висячих).

Tun $\{0\}$. Элементарные полипентагоны типа $\{0\}$ - это конечный полипентагон $A_{1}$ и бесконечный полипентагон $A_{6}$. Неэлементарные полипентагоны типа $\{0\}$ бесконечны и состоят из $C_{1}$ (смежных с двумя соседями), $C_{3}, E_{1}$ (смежных с тремя соседями).

Tun $\{3 ; 2 ; 1\}$. Полипентагоны типа $\{3 ; 2 ; 1\}$ неэлементарны и состоят из элементарных слагаемых $B_{1}, B_{2}, B_{3}, C_{1}, C_{2}, C_{3}, D, E_{j}$ при $j \geqslant 1$. Хотя бы одно $D$ висячее.

Tun $\{3 ; 2\}$. Полипентагоны типа $\{3 ; 2\}$ неэлементарны и состоят из элементарных слагаемых $C_{1}, C_{3}, D, E_{1}$. Каждое элементарное слагаемое $D$ является висячим.

Tun $\{3 ; 1\}$. Полипентагоны типа $\{3 ; 1\}$ неэлементарны и состоят из элементарных слагаемых $B_{1}, B_{2}, B_{3}, C_{1}, C_{2}, C_{3}, D, E_{j}$ при $j \geqslant 1$. Хотя бы одно $D$ является висячим.

Tun $\{2 ; 1\}$. Элементарные полипентагоны типа $\{2 ; 1\}$ суть $B_{1}, B_{2}, B_{3}, C_{2}, D, E_{j}$, $j \geqslant 2$. Неэлементарные полипентагоны типа $\{2 ; 1\}$ состоят из элементарных слагаемых $B_{1}, B_{2}, B_{3}, C_{1}, C_{2}, C_{3}, D, E_{j}, j \geqslant 1$. Каждое $D$ смежно с двумя соседями.

Любая пара эквиграничных полипентагонов типа $\{2 ; 1\}$ или $\{2\}$ приводима посредством следующей перестройки фрагмента граничного кода: $32^{2} 3 \rightarrow 232$.

Любая пара эквиграничных полипентагонов типа $\{3 ; 2 ; 1\},\{3 ; 2\},\{3 ; 1\}$ или $\{3\}$ приводима посредством перестройки фрагмента граничного кода: $32^{3} 3 \rightarrow 2^{2}$.

ЛЕмма 3. Полипентагоны неприводимой эквиграничной пары имеют тип $\{1\}$.

СледСтвиЕ. Эквиграничная пара с минималъным $p_{5}$ имеет mun $\{1\}$.

Среди элементарных слагаемых конечных неэлементарных полипентагонов типа $\{1\}$ есть висячие $B_{2}$ и/или $B_{3}$, могут быть не висячие $C_{1}$ и/или $C_{2}, C_{3}, D, E_{j}, j \geqslant 1$.

\section{Список литературы}

[1] M. Deza, M. Shtogrin, J. Geom. Phys., 40:3-4 (2002), 302-319. [2] X. Guo, P. Hansen, M. Zheng, Discrete Appl. Math., 118:3 (2002), 209-222. [3] G. Brinkmann, O. Delgado-Friedrichs, U. von Nathusius, Graphs and discovery, DIMACS Ser. Discrete Math. Theoret. Comput. Sci., 69, Amer. Math. Soc., Providence, RI, 2005, 27-38. [4] M. Dutour, M. Deza, M. Shtogrin, "Fillings of a given boundary by p-gons and related problems", Proceedings of International Conference "General Theory of Information Transfer and Combinatorics" (Bielefeld, 2004) (to appear).

М. Деза (M. Deza)

École Normale Supérieure, Paris

E-mail: Michel.Deza@ens.fr
Представлено В.М.Бухштабером Принято редколлегией 02.08.2006

М. И. Штогрин (М. I. Shtogrin)

Математический институт им. В. А. Стеклова РАН

E-mail: stogrin@mi.ras.ru 